\title{
Flexural Motions of Beams on Foundation Subjected to Moving Concentrated Force
}

\author{
Ogunyebi S.N., Adedowole A., Ogunlade T.O., Oyedele A. A.
}

\begin{abstract}
In this present paper, the dynamic analysis of nonprismastic beams subjected to moving concentrated forces is investigated at constant speed. Two cases of load-beam interaction problems described by the Dirac delta function with constant and harmonic magnitude mobile forces are studied. The technique called Galerkin's method in conjunction with integral transform method was employed to solve the motion equation. From the numerical results, it is evidently seen that an increase in the foundation stiffness provides reduction on the beam deflection. And furthermore, the issue of resonance is closely monitored and observed to have reached earlier in constant magnitude than harmonic variable magnitude problem. Results presented in this work are useful in constructions engineering designs.
\end{abstract}

Keywords: Vibrating, Non-Prismastic Beam, Concentrated forces, Harmonic load, foundation, Galerkin method.

\section{INTRODUCTION}

In the past years, problem of the vibrating motions of elastic structures (such as beam, plates, or shells) subjected to mobile forces has been given considerable attention by several researchers in Physics, Applied Mathematics and related fields. In particular, problem of uniform beams under the passage of moving loads has been studied by numerous authors [1,2,3] Dynamic motions of beam with non-uniformity when subjected to moving load was first addressed by Kolousek et al [4]. Their problems were solved using normal mode analysis. Sadiku and Leipholz [5] examined the dynamics of a prismatic beam and the effect of inertia was considered. Knowles [6] also worked on the dynamic deflection of a beam to a randomly moving load. The analysis of the expected beam deflection and bending moment was carried out when the load was moving with a variable velocity. The inertia effect of the load on the vibration of the system was not considered. Gutierrez and Laura [7] analysed the displacement response of a beam with a non-uniform cross-section and the approximate determination of the dynamic vibration of a beam on a time varying concentrated load. Masoud [8] examined vibration interaction analysis of non-prismatic cross-sectional beam structure under moving vehicle. The problem of a non-uniform cross-section beam with different boundary condition subjected to moving loads, such as a moving concentrated mass and a simple quarter-car (SQC) planar model was studied.

Revised Manuscript Received on February 05, 2020.

* Correspondence Author

Ogunyebi S. N*, Senior Lecturer, Department of Mathematics, Ekiti State University, Ado-Ekiti, Nigeria.

Adedowole A, Senior Lecturer, Department of Mathematical Sciences, Adekunle, Ajasin University, Akungba-Akoko, Nigeria.

Ogunlade, T. O, Senior Lecturer, Department of Mathematics, Ekiti State University, Ado-Ekiti, Nigeria.

Oyedele A. A, Senior Lecturer, Department of Physics, Ekiti State University, Ado-Ekiti, Nigeria.

(c) The Authors. Published by Blue Eyes Intelligence Engineering and Sciences Publication (BEIESP). This is an open access article under the CC BY-NC-ND license (http://creativecommons.org/licenses/by-nc-nd/4.0/)
These investigations though impressive, have neglected the more practical situation where the elastic structure are given as variable cross-section. The dynamic response of non-prismatic beam-type resting on variable elastic subgrade and traversed by moving concentrated masses is examined by Ogunyebi et al [9]. In the paper, the flexural effect of rotatory inertia is neglected. This paper presents the problem of vibration analysis of non-prismastic beam with exponential decaying foundation to mobile concentrated masses. The Galerkin's technique is used in the first instance to lower the order and simplify the motion equation from the fourth order to second order differential equations called Galerkin equations. Analytical solution will be of interest as it sheds light on vital information about the vibrating system.

\section{PROBLEM FORMULATION}

This section seeks the closed form solutions to the dynamical problem. The equation that governs the system subjected to moving load is given as Frybal [9]

$$
\begin{gathered}
E^{*} \frac{\partial^{4} y}{\partial x^{4}}+\mu(x) \frac{\partial^{2} y}{\partial x^{2}}+\varepsilon_{o} \frac{\partial y}{\partial t}+K(x) y=P(x, t) \\
+r_{o} \mu(x) \frac{\partial^{4} y}{\partial x^{2} \partial t^{2}}+N \frac{\partial^{2} y}{\partial x^{2}} \\
r_{o} \mu(x, t) \frac{\partial^{4} u(x, t)}{\partial x^{2} \partial t^{2}}=E I(x) \frac{\partial^{4} u(x, t)}{\partial x^{4}}+\mu(x) \frac{u(x, t)}{\partial x^{2}}+ \\
\varepsilon_{o} \frac{\partial u(x, t)}{\partial t}+K(x) u(x, t)
\end{gathered}
$$

where, ${ }^{y=u(x, t) E^{*}=E I(x)}{ }_{x} x \quad$ represent spacial coordinate, $t$ the time, $u(x, t)^{y}$ the Displacement, $E E$ the Young's modulus, $\mu(x)^{\mu(x)}$ the mass, $I(x)$ the inertia of the beam, $K(x)$ is the variable elastic foundation and $r_{o} r_{o}$ is the measure of rotating inertia.

Defining moment of inertia (variable type) and the mass of the beam to be

For the variable moment of inertia $I I$ and the mass per unit length $\mu^{\mu}$ of the beam adopt the example in [8] and take $I(x) I(x)$ and $\mu(x)$ to be of the form,

$$
\begin{aligned}
& I(x)=I_{o}\left(1+D_{o}\right)^{3} I(x)= \\
& I_{\mathrm{o}}\left(1+\sin \frac{\pi x}{L}\right)^{3}
\end{aligned}
$$

and

$$
\mu(x)=\mu_{o}\left(1+D_{o}\right)
$$


where

$$
\mu(x)=\mu_{\mathrm{o}}(1+\sin \pi x / L)
$$

$$
D_{o}=\sin \frac{\pi x}{L}
$$

The rigidity of exponential form is given by

$$
\begin{aligned}
& K(x)=K_{\mathrm{o}} e^{-\lambda x} \\
& K(x)=K_{0} e^{-\lambda x}
\end{aligned}
$$

where $\lambda$ is a constant and $K_{0}$ is the elastic foundation constant.

\section{BEAM ON EXPONENTIAL DECAY FOUNDATION UNDER CONSTANT MAGNITUDE}

In this section, we define

$$
\begin{aligned}
& P(x, t)=P_{o} \delta\left(x-c_{m} t\right) \\
P(x, t)= & P_{\mathrm{o}} \delta\left(x-c_{m} t\right)
\end{aligned}
$$

where $c_{m}$ is the velocity of the $m^{t h} m^{t h}$ particles of the system. Equation (3.0) describe the mobile concentrated load and I is taken to be of constant magnitude

Putting equations (2.2), (2.3), (2.4) and (3.0) are substituted into equation (2.0), one obtains

$$
\begin{aligned}
& E I_{o} \frac{\partial^{2}}{\partial x^{2}}\left[\left(1+\sin \frac{\pi x}{L}\right)^{3} \frac{\partial^{2} u(x, t)}{\partial x^{2}}\right]+\mu_{o}\left(1+\sin \frac{\pi x}{L}\right) \frac{\partial^{2} u(x, t)}{\partial t^{2}} \\
& -r_{o} \mu_{o}\left(1+\sin \frac{\pi x}{L}\right) \frac{\partial^{4} u(x, t)}{\partial x^{2} \partial t^{2}} \\
& +\varepsilon_{o} \frac{\partial u(x, t)}{\partial t}-N \frac{\partial^{2} u(x, t)}{\partial x^{2}}+K_{0} e^{-\lambda x} u(x, t) \\
& =P_{o} \delta\left(x-c_{m} t\right)
\end{aligned}
$$

\section{METHOD OF SOLUTION}

The versatile solution technique called Galerkin's method to solve the beam problem above is employed. To this end, it is given in the form

$\theta(u)-P_{\mathrm{o}}=0 \theta u(x, t)-P_{0}(x, t)=0$

Where $\theta$ represent differential operator, $\mathrm{u}$ the structural deflection and $\mathrm{P}_{\mathrm{o}}$ the load on the beam. The solution of the beam problem is expressed as

$$
y=\sum_{i=1}^{n} P_{i} Q_{i}
$$

$$
u(x, t)=\Sigma Y_{i}(t) H_{i}(x)
$$

where $P_{i}$ and $Q_{i}$ are functions of $\mathrm{t}$ and $\mathrm{x}$ respectively. ${ }{ }_{i}$ is given as modal coordinates and $Q_{i}$ is

$Q_{i}(x)=\sin \alpha_{a}+A_{i} \cos \alpha_{a}+B_{i} \sinh \alpha_{a}+C_{i} \cosh \alpha_{a}$ $C_{i} \cosh \alpha_{i} x(3.4)$

$$
Q_{i}(x)=\sin \alpha_{i} x+A_{i} \cos \alpha_{i} x+B_{i} \sinh \alpha_{i} x+
$$

where the constant $A_{i}, B_{i}$, and $C_{i}$ are amplitude of vibration and $\alpha_{a}=\alpha_{i} x$.

Noting that the problem at hand is simply supported, equation (3.3) upon consideration of equation (3.4) can be re-written in the form

$$
\begin{aligned}
& y=\sum_{i=1}^{\infty} P_{i} \sin \frac{i \pi x}{L} \\
& u \_a(x, t)=\sum_{-}(i=
\end{aligned}
$$

$1)^{\wedge} \infty P_{-} i(t) \sin i \pi x / L(3.5)$

Putting equation (3.5) above into equation (3.1) gives $\frac{E I_{o}}{4}\left[\left(10-6 \cos \frac{\pi x}{L}+15 \sin \frac{\pi x}{L}-\sin \frac{3 \pi x}{L}\right) \frac{\partial^{4}}{\partial x^{4}} \sum_{i=1}^{\infty} P_{i}(t) \sin \frac{i \pi x}{L}\right]$ $+\frac{3 \pi}{L}\left(\sin \frac{3 \pi x}{L}+5 \cos \frac{\pi x}{L}-\cos \frac{3 \pi x}{L}\right)$ $\frac{\partial^{3}}{\partial x^{3}} \sum_{i=1}^{\infty} P_{i}(t) \sin \frac{i \pi x}{L} 3\left(\frac{\pi}{L}\right)^{2}$ $\left(8 \cos \frac{2 \pi x}{L}-5 \sin \frac{\pi x}{L}+3 \sin \frac{3 \pi x}{L}\right) \frac{\partial^{2}}{\partial x^{2}} \sum_{i=1}^{\infty} P_{i}(t) \sin \frac{i \pi x}{L}$

$+\mu_{o}\left(1+\sin \frac{\pi x}{L}\right) \frac{\partial^{2}}{\partial x^{2}} \sum_{i=1}^{n} P_{i}(t) \sin \frac{i \pi x}{L}$

$-r_{o} \mu_{o}\left(1+\sin \frac{\pi x}{L}\right) \frac{\partial^{4}}{\partial x^{2} \partial t^{2}} \sum_{i=1}^{n} P_{i}(t) \sin \frac{i \pi x}{L}$

$+\varepsilon_{o} \frac{\partial}{\partial t} \sum_{i=1}^{n} P_{i}(t) \sin \frac{i \pi x}{L}-N \frac{\partial^{2}}{\partial x^{2}} \sum_{i=1}^{n} P_{i}(t) \sin \frac{i \pi x}{L}$

$+K_{0} e^{-\lambda x} \sum_{i=1}^{n} P_{i}(t) \sin \frac{i \pi x}{L}=P_{o} \delta\left(x-c_{i} t\right)$

$\frac{E I_{\mathrm{o}}}{4}\left[\left(10-6 \cos \cos \frac{2 \pi x}{L}+15 \sin \frac{\pi x}{L}-\right.\right.$

$\left.\sin \frac{3 \pi x}{L}\right) \frac{\partial^{4}}{\partial x^{4}} \sum_{i=1}^{n} \quad P_{i}(t) \sin \frac{i \pi x}{L}++\frac{3 \pi}{L}\left(4 \sin \frac{2 \pi x}{L}+\right.$

$\left.5 \cos \frac{\pi x}{L}-\cos \frac{3 \pi x}{L}\right) \frac{\partial^{3}}{\partial x^{3}} \sum_{i=1}^{n} \quad P_{i}(t) \sin \frac{i \pi x}{L}+$

$3\left(\frac{\pi}{L}\right)^{2}\left(8 \cos \frac{2 \pi x}{L}-5 \sin \frac{\pi x}{L}+\right.$

$\left.\left.3 \sin \frac{3 \pi x}{L}\right) \frac{\partial^{2}}{\partial x^{2}} \sum_{i=1}^{n} \quad P_{i}(t) \sin \frac{i \pi x}{L}\right]+\mu_{0}(1+$ $\left.\sin \frac{\pi x}{L}\right) \frac{\partial^{2}}{\partial t^{2}} \sum_{i=1}^{n} \quad P_{i}(t) \sin \frac{i \pi x}{L}--r_{0} \mu_{0}(1+$ $\left.\sin \frac{\pi x}{L}\right) \frac{\partial^{4}}{\partial x^{2} \partial t^{2}} \sum_{i=1}^{n} \quad P_{i}(t) \sin \frac{i \pi x}{L}+$

$\varepsilon_{0} \frac{\partial}{\partial t} \sum_{i=1}^{n} \quad P_{i}(t) \sin \frac{i \pi x}{L}+K_{0} e^{-\lambda x} \sum_{i=1}^{n} \quad P_{i}(t) \sin \frac{i \pi x}{L}=$ $P_{0} \delta\left(x-c_{i} t\right)$

Subjecting equation (3.6) to further simplification, one obtains

$\frac{E I_{o}}{4}\left[R_{1}(x)\left(A_{1}\right)^{4} \sin \phi x-R_{2}(x)\left(A_{1}\right)^{3} \cos \phi x-R_{3}(x)\left(A_{1}\right)^{2} \sin \phi x\right] P_{i}(t)$

$+\mu_{o} R_{4}(x) \ddot{P}_{i}(t) \sin \phi x+(\phi)^{2} r_{o} \mu_{o} R_{4}(x) \ddot{P}_{i}(t) \sin \phi x+\varepsilon_{o} P_{i}(t) \sin \phi x$

$+N(\phi)^{2} P_{i}(t) \sin \phi x+K_{0} e^{-\lambda x} P_{i}(t) \sin \phi x=P_{o} \delta\left(x-c_{i} t\right)$

where

$$
\begin{aligned}
& A_{1}=\frac{i \pi x}{L}, A_{2}=\frac{\sin \pi x}{L} \\
& \phi=\frac{i \pi}{L}
\end{aligned}
$$

and

$$
R_{1}(x)=\left(10-6 \cos \frac{\pi x}{L}+15 \sin \frac{\pi x}{L}-\sin \frac{3 \pi x}{L}\right)
$$




$$
\begin{aligned}
& R_{1}(x)=10-6 \cos \frac{2 \pi x}{L}+15 \sin \frac{\pi x}{L}-\sin \frac{3 \pi x}{L} \\
& R_{2}(x)=\frac{3 \pi}{L}\left(\sin \frac{3 \pi x}{L}+5 \cos \frac{\pi x}{L}-\cos \frac{3 \pi x}{L}\right) \\
& R_{2}(x)=\frac{3 \pi}{2}\left(4 \sin \frac{2 \pi x}{L}+5 \cos \frac{\pi x}{L}-\cos \frac{3 \pi x}{L}\right) \\
& R_{3}(x)=3\left(\frac{\pi}{L}\right)^{2}\left(8 \cos \frac{2 \pi x}{L}-5 \sin \frac{\pi x}{L}+3 \sin \frac{3 \pi x}{L}\right) \\
& R_{4}(x)=\left(1+\sin \frac{\pi x}{L}\right)
\end{aligned}
$$

By the imposition of orthogonality conditions, equation (3.7) becomes

$$
\begin{aligned}
& \int_{0}^{L} \sum_{i=1}^{n}\left\{\frac{E I_{o}}{4}\left[\begin{array}{c}
\left.R_{1}(x)\left(\frac{i \pi x}{L}\right)^{4} \sin \frac{i \pi x}{L}-R_{2}(x)\left(\frac{i \pi x}{L}\right)^{3}\right] \\
\cdot \cos \frac{i \pi x}{L}-R_{3}(x)\left(\frac{i \pi x}{L}\right)^{2} \sin \frac{i \pi x}{L}
\end{array}\right] P_{i}(t)\right. \\
& +\mu_{o} R_{4}(x) \ddot{P}_{i}(t) \sin \frac{i \pi x}{L} \\
& +\left(\frac{i \pi}{L}\right)^{2} r_{o} \mu_{o} R_{4}(x) \ddot{P_{i}}(t) \sin \frac{i \pi x}{L} \\
& +N\left(\frac{i \pi}{L}\right)^{2} P_{i}(t) \sin \frac{i \pi x}{L}+\varepsilon_{o} P_{i}(t) \sin \frac{i \pi x}{L} \\
& \left.+K_{0} e^{-\lambda x} P_{i}(t) \sin \frac{i \pi x}{L}\right\} \sin \frac{k \pi x}{L}=\int_{0}^{l} P_{o} \delta\left(x-c_{i} t\right) \sin \frac{k \pi x}{L}
\end{aligned}
$$

Equation (3.10) after some rearrangements and simplifications yields

$$
D_{1}(i, k) \frac{\mathbb{P}}{i}(t)+D_{2}(i, k) D_{i}(t)+D_{3}(i, k) P_{i}(t)=P_{o} \sin \frac{k \pi c t}{L}
$$

where

$$
\begin{aligned}
& \left.D_{1}(i, k)=\mu_{o}\left[I_{1}+I_{3}\right] 1+\left(\frac{i \pi}{L}\right)^{2} r_{o}\right] \\
& D_{2}(i, k)=\varepsilon_{o} I_{1} D_{2}=\varepsilon_{0} I_{1} \\
& \quad(3.12 \mathrm{~b}) \\
& D_{3}(i, k)=\frac{E I_{o}}{4}\left[E_{1}-E_{2}-E_{3}\right]+E_{4} D_{3}=\frac{E I_{0}}{4}\left[E_{1}-E_{2}-\right. \\
& \left.E_{3}\right]+E_{4}(3.12 \mathrm{c}) \\
& E_{1}=\left(\frac{i \pi}{L}\right)^{4}\left[10 I_{1}-6 I_{2}+15 I_{3}-I_{4}\right] \\
& \left.6 I_{2}+15 I_{3}-I_{4}\right](3.13) \\
& E_{2}=3 i^{3}\left(\frac{\pi}{L}\right)^{4}\left[4 I_{5}+5 I_{6}-I_{7}\right]
\end{aligned}
$$

$$
\begin{aligned}
& E_{3}=3 i^{2}\left(\frac{\pi}{L}\right)^{4}\left[8 I_{2}-5 I_{3}+3 I_{4}\right] E_{3}=3 i^{2}\left(\frac{\pi}{L}\right)^{4}\left[8 I_{2}-\right. \\
& \left.5 I_{3}+3 I_{4}\right]
\end{aligned}
$$

The integrals $I_{i}$ are as follow

$$
\begin{aligned}
& I_{1}=\int_{0}^{L} \sin \frac{i \pi x}{L} \sin \frac{k \pi x}{L} d x,_{I_{1}}= \\
& \int_{0}^{L} \operatorname{Sin} \frac{i \pi x}{L} \operatorname{Sin} \frac{k \pi x}{L} d x \\
& I_{2}=\int_{0}^{L} \cos \frac{2 \pi x}{L} \sin A_{1} \sin A_{3} d x \\
& I_{2}=\int_{0}^{L} \quad \operatorname{Cos} \frac{2 \pi x}{L} \operatorname{Sin} \frac{i \pi x}{L} \sin \frac{k \pi x}{L} d x \\
& I_{3}=\int_{0}^{L} \sin \frac{\pi x}{L} \sin A_{1} \sin A_{3} d x I_{3}= \\
& \int_{0}^{L} \operatorname{Sin} \frac{\pi x}{L} \operatorname{Sin} \frac{i \pi x}{L} \operatorname{Sin} \frac{k \pi x}{L} d x \\
& I_{4}=\int_{0}^{L} \sin \frac{3 \pi x}{L} \sin A_{1} \sin A_{3} d x \\
& I_{4}=\int_{0}^{L} \quad \operatorname{Sin} \frac{3 \pi}{L} \operatorname{Sin} \frac{i \pi x}{L} \operatorname{Sin} \frac{k \pi x}{L} d x \\
& I_{5}=\int_{0}^{L} \sin \frac{2 \pi x}{L} \cos \frac{i \pi x}{L} \sin \frac{k \pi x}{L} d x \\
& I_{6}=\int_{0}^{L} \cos \frac{\pi x}{L} \cos \frac{i \pi x}{L} \sin \frac{k \pi x}{L} d x I_{I_{5}}= \\
& \int_{0}^{L} \operatorname{Sin} \frac{2 \pi x}{L} \operatorname{Cos} \frac{i \pi x}{L} \operatorname{Sin} \frac{k \pi x}{L} d x \\
& I_{7}=\int_{0}^{L} \cos \frac{3 \pi x}{L} \cos \frac{i \pi x}{L} \sin \frac{k \pi x}{L} d x \\
& A_{3}=\frac{k \pi x}{L}
\end{aligned}
$$

Evaluating the integrals in (3.16a), equations (3.12a) to (3.15) yield the following respectively

$$
E_{1}=\left(\frac{i \pi}{L}\right)^{4}\left[\begin{array}{l}
5 L+\frac{3 i L}{2}-\frac{60 i k L}{\pi\left[1-(i-k)^{2}\right]\left[1-(i+k)^{2}\right]} \\
+\frac{12 i k L}{\pi\left[9-(i-k)^{2}\right]\left[9-(i+k)^{2}\right]}
\end{array}\right]
$$$$
E_{2}=3 i^{3}\left(\frac{\pi}{L}\right)^{4}\left[\begin{array}{c}
k L-\frac{60 i k L\left[1+i^{2}+k^{2}\right]}{\pi\left[1-(i-k)^{2}\right]\left[1-(i+k)^{2}\right]} \\
+\frac{2 k L\left[9+i^{2}-k^{2}\right]}{\pi\left[9-(i-k)^{2}\right]\left[9-(i+k)^{2}\right]}
\end{array}\right]_{(3.18)}
$$$$
E_{3}=3 i^{2}\left(\frac{\pi}{L}\right)^{4}\left[-2 i L-\frac{20 i k L}{\pi\left[1-(i-k)^{2}\left[1-(i+k)^{2}\right]\right.}+\frac{36 i k L}{\pi\left[9-(i-k)^{2}\left[9-(i+k)^{2}\right]\right.}\right]
$$

And 
$E_{4}=K_{o}\left(\frac{(\pi(i-k) / L)}{2\left[(i-k)^{2}\left(\frac{\pi}{L}\right)^{2}+\lambda^{2}\right]}\right)\left[e^{-\lambda L} \sin \pi(i-k)-\frac{e^{-\lambda L} \cos \pi(i-k)}{(\pi(i-k) / L)}+\frac{\lambda}{(\pi(i-k) / L)}\right]$

$K_{o}\left(\frac{(\pi(i+k) / L)}{2\left[(i+k)^{2}\left(\frac{\pi}{L}\right)^{2}+\lambda^{2}\right]}\right)$

$\left[e^{-\lambda L} \sin \pi(i+k)-\frac{e^{-\lambda L} \cos \pi(i+k)}{(\pi(i+k) / L)}+\frac{\lambda}{(\pi(i+k) / L)}\right]$

$E_{4}=K_{0}\left\{\frac{(i-k) \frac{\pi}{L}}{2\left[(i-k)^{2}\left(\frac{\pi}{L}\right)^{2}+\lambda^{2}\right]}\left[e^{-\lambda L} \quad \operatorname{Sin}(i-k) \pi-\right.\right.$

$\frac{\lambda e^{-\lambda L} \operatorname{Cos}(i-k) \pi}{(i-k) \frac{\pi}{2}}+\frac{\lambda}{\left.(i-k) \frac{\pi}{L}\right]}-\frac{(i+k) \frac{\pi}{L}}{2\left[(i+k)^{2}\left(\frac{\pi}{L}\right)^{2}+\lambda^{2}\right]} \times\left[e^{-\lambda L} \operatorname{Sin}(i+\right.$

$\left.\left.k) \pi-\frac{\lambda e^{-\lambda L} \operatorname{Cos}(i+k) \pi}{(i-k) \frac{\pi}{L}}+\frac{\lambda}{(i+k) \frac{\pi}{L}}\right]\right\}$

At this stage, equation (3.11) is subjected to a Laplace transform and after further simplification give

$\left(D_{1}(i, k) S^{2}+D_{2}(i, k) S+D_{3}(i, k)\right) P_{i}(s)$

$=P_{0} \frac{S}{S^{2}+\theta^{2}}$

where

$$
\theta=\left(\frac{k \pi c_{i}}{L}\right) \quad \theta=\frac{k \pi c_{i}}{L}
$$

Subjecting equation (3.21) to some simplifications and rearrangements gives

$P_{i}(s)=P_{0} \frac{\theta}{S^{2}+\theta^{2}} \frac{1}{\left(D_{1}(i, k) S^{2}+D_{2}(i, k) S+D_{3}(i, k)\right)}$

which reduces to

$$
\begin{aligned}
& P_{i}(S)=\frac{P_{0}}{\left(\beta_{1}-\beta_{2}\right)}\left(\frac{S}{S^{2}+\theta^{2}} \cdot \frac{1}{S-\beta_{1}}-\frac{S}{S^{2}+\theta^{2}} \cdot \frac{1}{S-\beta_{2}}\right) \\
& P_{i(s)}=\frac{P_{0}}{\left(\beta_{1}-\beta_{2}\right)} \cdot \frac{\theta}{S^{2}+\theta^{2}}\left(\frac{1}{S-\beta_{1}}-\frac{1}{S-\beta_{2}}\right)
\end{aligned}
$$

where

$$
\begin{aligned}
& \beta_{1}=\frac{-D_{2}+\sqrt{D_{2}^{2}-4 D_{1} D_{3}}}{2 D_{1}} \\
& \frac{-D_{2}+\sqrt{D_{2}^{2}-4 D_{1} D_{3}}}{2 D_{1}} \text { and } \beta_{2}=\frac{-D_{2}-\sqrt{D_{2}^{2}-4 D_{1} D_{3}}}{2 D_{1}}
\end{aligned}
$$

and

$$
\beta_{2}=\frac{-D_{2}-\sqrt{D_{2}^{2}-4 D_{1} D_{3}}}{2 D_{1}}
$$

Adopting the representation below, the Laplace inversion of equation (3.24) is given as

$$
\begin{aligned}
& g(s)=\frac{\theta}{S^{2}+\theta^{2}}, f_{1}(s)=\frac{1}{S-\beta_{1}} \\
& f_{2}(s)=\frac{1}{S-\beta_{2}}
\end{aligned}
$$

Further simplification and arrangements of (3.26) gives $P_{i}(t)=P_{p}\left[\frac{e^{\beta_{1} t}}{\beta_{1}} I_{1}-\frac{e^{\beta_{2} t}}{\beta_{2}} I_{2}\right]$

where

$P_{p}=\frac{P_{0}}{\left(\beta_{1}+\beta_{2}\right)} P_{p}=\frac{P_{0}}{\beta_{1}+\beta_{2}}$
$I_{1}=\int_{0}^{t} e^{-\beta_{1} u} \sin \theta u d u$ and

$I_{1}=\int_{0}^{t} e^{-\beta_{1} u} \sin \theta u d u$ and $I_{2}=\int_{0}^{t} e^{-\beta_{2} u} \sin \theta u d u$

Evaluating the integrals (3.29) above, we obtain $I_{1}=\frac{1}{\left(\theta^{2}+\beta_{1}^{2}\right)}\left(-\theta e^{-\beta_{1} t} \cos \theta t+\theta-\beta_{1} e^{-\beta_{1} t} \sin \theta t\right)$

$I_{2}=\frac{1}{\left(\theta^{2}+\beta_{2}^{2}\right)}\left\{-\theta e^{-\beta_{2} t} \cos \cos \theta t+\theta-\beta_{2} e^{-\beta_{2} t}\right.$ $\sin \sin \theta t\}$

$I_{2}=\frac{1}{\left(\theta^{2}+\beta_{2}^{2}\right)}\left(-\theta e^{-\beta_{2} t} \cos \theta t+\theta-\beta_{2} e^{-\beta_{2} t} \sin \theta t\right)$

Subjecting equation (3.27) to some simplification and rearrangement yields,

$$
\begin{aligned}
& P_{i}(t)=\frac{P_{p}}{\beta_{1}\left(\theta^{2}+\beta_{1}^{2}\right)}\left(\theta\left(e^{\beta_{1} t}-\cos \theta t\right)-\beta_{1} \sin \theta t\right) \\
& -\frac{P_{p}}{\beta_{2}\left(\theta^{2}+\beta_{2}^{2}\right)}\left(\theta\left(e^{\beta_{2} t}-\cos \theta t\right)-\beta_{2} \sin \theta t\right)
\end{aligned}
$$

which on invasion yields

$$
\begin{aligned}
& u(x, t)=\sum_{i=1}^{n}\left\{\frac{P_{p}}{\beta_{1}\left(\theta^{2}+\beta_{1}^{2}\right)}\left(\theta\left(e^{\beta_{1} t}-\cos \theta t\right)-\beta_{1} \sin \theta t\right)\right. \\
& \left.-\frac{P_{p}}{\beta_{2}\left(\theta^{2}+\beta_{2}{ }^{2}\right)}\left(\theta\left(e^{\beta_{2} t}-\cos \theta t\right)-\beta_{2} \sin \theta t\right)\right\} \sin \frac{i \pi x}{L} \\
& u(x . t)=\sum_{i=1}^{n}\left\{\frac { p _ { p } \beta _ { 1 } } { \theta ^ { 2 } + \beta _ { 1 } { } ^ { 2 } } \left[\theta\left(e^{\beta_{1} t}-\cos \cos \theta t\right)-\right.\right. \\
& \left.\sin \beta_{1} \sin \theta t\right]-\frac{p_{p \beta_{2}}}{\left(\theta^{2}+\beta_{2}{ }^{2}\right)}\left[\theta\left(e^{\beta_{2} t}-\beta_{2} \cos \cos \theta t\right)-\right. \\
& \left.\left.\sin \beta_{2} \sin \theta t\right]\right\} \sin \sin \frac{i \pi x}{L}
\end{aligned}
$$

which is the solution of the governing differential equation of the non-prismatic element at constant mobile distributed masses on exponential decaying subgrade. 


\section{BEAM ON EXPONENTIAL DECAY FOUNDATION UNDER HARMONIC MAGNITUDE}

This section seeks the solution of the non-prismatic structure with exponentially decaying foundation under harmonic magnitude at constant speed. The load on the structure is given as

$$
P(x, t)=P_{o} \cos \omega t \delta\left(x-c_{m} t\right){ }_{P(x, t)}=
$$

$P \cos \omega t \delta\left(x-c_{m} t\right)$

Using equation (2.0) in (4.1), the differential equation gives

$$
\begin{gathered}
E I(x) \frac{\partial^{4} u_{a}(x, t)}{\partial x^{4}}+\mu(x) \frac{\partial^{2} u_{a}(x, t)}{\partial x^{2}}-r_{o} \mu(x) \frac{\partial^{4} u_{a}(x, t)}{\partial x^{2} \partial t^{2}}+\varepsilon_{o} \frac{\partial u_{a}(}{\partial} \\
+K(x) u_{a}(x, t)=P_{o} \cos \omega t \delta\left(x-c_{m} t\right) \\
u_{a}(x, t)=\sum_{m=1}^{\infty} P_{m}(t) Q_{m}(x) \\
\sum_{m=1}^{\infty} \quad P_{m}(t) H_{m}(x)
\end{gathered}
$$

And for the simply supported study, equation (4.3) is rewritten in the form

$$
u_{a}(x, t)=\sum_{m=1}^{\infty} P_{m}(t) \sin \frac{m \pi x}{L} \quad u_{b}(x, t)=
$$

$\sum_{m=1}^{\infty} \quad P_{m}(t) \sin \frac{m \pi x}{L}$

Putting equation (4.4) into equation (4. 2) and using the same procedures as in section (3.0) one obtains

$$
\sum_{m=1}^{\infty}\left\{D_{1} \frac{d^{2} P_{m}}{d t^{2}}+D_{2} \frac{d P_{m}}{d t}+D_{3} P_{m}\right\}=P_{o} \cos \omega t \sin \frac{k \pi c_{m} t}{L}
$$

where $c_{m} c_{m}$ is the speed and $P_{m}(t)$.

Considering the mth particle of the system under consideration, equation (4.5) becomes

$D_{1} \frac{d^{2} P_{m}}{d t^{2}}+D_{2} \frac{d P_{m}}{d t}+D_{3} P_{m}=P_{o} \cos \omega t \sin \theta$

Subjecting equation (4.6) as defined previously yields

$$
y_{m}(S)=\frac{P_{o}}{2\left(d_{1}-d_{2}\right)}\left\{\frac{1}{\alpha_{1}}\left(\frac{\Omega_{1}}{S^{2}+\Omega_{1}^{2}} \cdot \frac{\alpha_{1}}{S-\alpha_{1}}-\frac{\Omega_{2}}{S^{2}+\Omega_{2}^{2}} \cdot \frac{\alpha_{1}}{S-\alpha_{1}}\right)\right.
$$

$$
-\frac{1}{\alpha_{2}}\left(\frac{\Omega_{1}}{S^{2}+\Omega_{1}^{2}} \cdot \frac{\alpha_{2}}{S-\alpha_{2}}-\frac{\Omega_{2}}{S^{2}+\Omega_{2}^{2}} \cdot \frac{\alpha_{2}}{S-\alpha_{2}}\right)
$$

where

$$
\begin{aligned}
& \Omega_{1}=\omega+\frac{k \pi c_{m}}{L} \quad \Omega_{2}=\omega-\frac{k \pi c_{m}}{L} \\
& \alpha_{1}=\frac{-D_{2}+\sqrt{D_{2}^{2}-4 D_{1} D_{3}}}{2 D_{1}} \\
& \frac{-D_{2}+\sqrt{D_{2}^{2}-4 D_{1} D_{3}}}{2 D_{1}} \text { and } \beta_{2}=\frac{-D_{2}-\sqrt{D_{2}^{2}-4 D_{1} D_{3}}}{2 D_{1}}
\end{aligned}
$$

and

$$
\alpha_{2}=\frac{-D_{2}-\sqrt{D_{2}^{2}-4 D_{1} D_{3}}}{2 D_{1}}
$$

Following the argument in section (3.0), equation (4.6) gives

$$
\begin{aligned}
& P_{m}(t)=\frac{P_{p}}{\beta_{1}\left(\Omega_{1}^{2}+\beta_{1}^{2}\right)}\left(\Omega_{1}\left(e^{\beta_{1} t}-\cos \Omega_{1} t\right)-\beta_{1} \sin \Omega_{1} t\right) \\
& -\frac{P_{p}}{\beta_{1}\left(\Omega_{2}^{2}+\beta_{1}^{2}\right)}\left(\Omega_{2}\left(e^{\beta_{1} t}-\cos \Omega_{2} t\right)-\beta_{1} \sin \Omega_{2} t\right) \\
& -\frac{P_{H} \alpha_{2}}{\left(\Omega_{1}^{2}+\alpha_{2}^{2}\right)}\left\{\Omega_{1}\left(e^{\alpha_{2} t}-\cos \Omega_{1} t\right)-\alpha_{2} \sin \Omega_{1} t\right\}+ \\
& \frac{P_{H} \alpha_{2}}{\left(\Omega_{2}^{2}+\alpha_{2}^{2}\right)}\left\{\Omega_{2}\left(e^{\alpha_{2} t}-\cos \Omega_{2} t\right)-\alpha_{2} \sin \Omega_{2} t\right\} \\
& -\frac{P_{p}}{\alpha_{2}\left(\alpha_{1}^{2}+\alpha_{2}^{2}\right)}\left(\Omega_{1}\left(e^{\alpha_{2} t}-\cos \Omega_{1} t\right)-\alpha_{2} \sin \Omega_{1} t\right) \\
& +\frac{P_{p}}{\alpha_{2}\left(\Omega_{2}^{2}+\alpha_{2}^{2}\right)}\left(\Omega_{2}\left(e^{\alpha_{2} t}-\cos \Omega_{2} t\right)-\alpha_{2} \sin \Omega_{2} t\right)
\end{aligned}
$$

which on inversion yields

$$
\begin{aligned}
& u_{b}(x, t)=\sum_{m=1}^{n}\left[\frac{P_{p}}{\alpha_{1}\left(\Omega_{1}^{2}+\alpha_{1}^{2}\right)}\left(\Omega_{1}\left(e^{\alpha_{1} t}-\cos \Omega_{1} t\right)-\alpha_{1} \sin \Omega_{1} t\right)\right. \\
& -\frac{P_{p}}{\alpha_{1}\left(\Omega_{2}^{2}+\alpha_{1}^{2}\right)}\left(\Omega_{2}\left(e^{\alpha_{1} t}-\cos \Omega_{2} t\right)-\alpha_{1} \sin \Omega_{2} t\right) \\
& -\frac{P_{p}}{\alpha_{2}\left(\alpha_{1}^{2}+\alpha_{2}^{2}\right)}\left(\Omega_{1}\left(e^{\alpha_{2} t}-\cos \Omega_{1} t\right)-\alpha_{2} \sin \Omega_{1} t\right) \\
& \left.+\frac{P_{p}}{\alpha_{2}\left(\Omega_{2}^{2}+\alpha_{2}^{2}\right)}\left(\Omega_{2}\left(e^{\alpha_{2} t}-\cos \Omega_{2} t\right)-\alpha_{2} \sin \Omega_{2} t\right)\right] \sin \frac{m \pi x}{L}
\end{aligned}
$$

which is the deflection response of the structure subjected to actions of harmonic magnitude load at constant velocity.

\section{DISCUSSION}

\section{Effects of Resonance}

The dynamic effect of resonance conditions is paramount in the study of dynamic system such as beam problem. These are the conditions under which the non-prismatic beam response grow without bound.

In studying a dynamic problem such as this, it is desirable to examine the phenomenon of resonance condition. These are the conditions under which the non- prismastic beam responses grow without bound.

Evidently, equation (3.34) shows that the non- prismastic beam response under constant magnitude mobile concentrated forces will grow without bound whenever

$$
\beta_{1}=\beta_{2}, \quad \theta^{2}=-\beta_{1}^{2} \text { or } \quad \theta^{2}=-\beta_{2}^{2}
$$

And the velocity at which this occurs is termed the critical condition, while from equation (4.8) the same nonprismastic beam traversed by harmonic variable magnitude mobile forces will experience resonance effects whenever

$$
\alpha_{1}=\alpha_{2}, \Omega_{1}^{2}=-\alpha_{1}^{2}, \text { or } \Omega_{2}^{2}=-\alpha_{2}^{2}
$$

It can be deduced by equations (5.1) and (5.2) that the critical velocity of the structure when on exponentially decaying subgrade is smaller than when the system involving non-pragmatic beam subjected to harmonic variable magnitude mobile force. Therefore, resonance is reached earlier in constant magnetic than harmonic variable magnitude. 


\section{NUMERICAL CALCULATIONS AND DISCUSSION OF RESULTS}

As an illustration, the non-prismatic beam is taken to be $12.192 \mathrm{~m}$ long and the mass is taken to travel at the constant velocity $8.12 \mathrm{~m} / \mathrm{s}$. Furthermore, we assumed that force travels at the EI is $6068242 \mathrm{~m}^{2} / \mathrm{s}^{2}$

$\mu=2758.291 \mathrm{~kg} / \mathrm{m}$. The deflection of the beam with exponential decaying foundation for the values of $\mathrm{K}$ and $\mathrm{R}_{0}$ are plotted against t. The analysis was carried out separately for both cases of constant magnitude and harmonic magnitude of mobile forces problems.

Figure 1 displays the effect of foundation modulus $K_{o} K_{0}$ on the transverse deflection of non-prismastic elastic beam when traversing concentrated forces are of constant magnitudes. The figure depict that as $K_{o}$ increases the displacement response of beam decreases.

Figure 2 depicts the deflection of FM for constant force for fixed value of Rotatory inertia $\mathrm{R}_{0}$ and axial force $\mathrm{N}$. It is seen that an increase in FM reduces the deflection profile of the beam.

Figure 3 Figure 3 shows deflection of axial force on the transverse deflection of non-prismatic elastic beam when traversing concentrated forces is of constant magnitudes. An increase in $\mathrm{N}$ decreases the deflection of the elastic beam.

Figure 4 displays the response amplitude of the harmonic mobile forces. It is clearly seen the response amplitude decreases as rotatory inertia increases.

Figure 5 displays the deflection of the harmonic mobile forces for the beam. The response amplitude decreases as the FM of the beam increases.

Figure 6 displays the response amplitude of harmonic mobile forces for the beam. The response amplitude decreases as axial force $\mathrm{N}$ increases.

Figure 7 compares the deflection profiles of the nonprismatic structure for constant magnitude and harmonic magnitude at constant speed for fixed values of $\mathrm{FM}=4000000, \mathrm{~N}=, 500000 K_{o} 0.5$. Evidently, the deflection amplitude of constant magnitude mobile load is higher than that of the harmonic variable mobile forces.

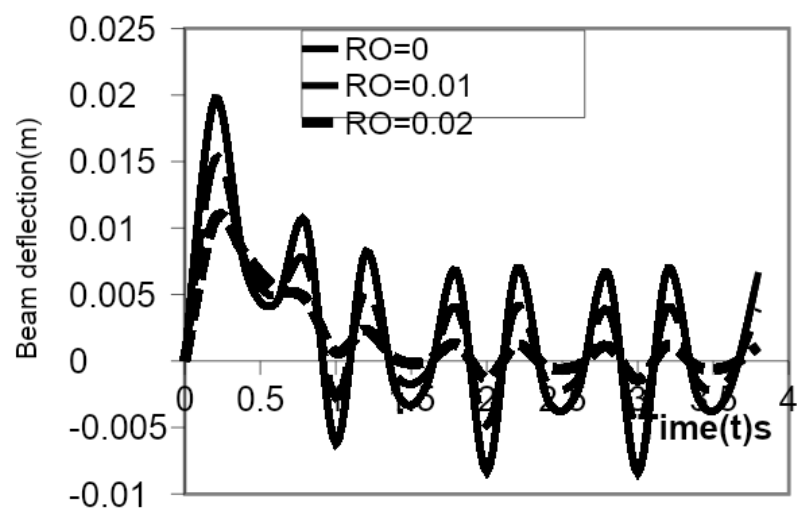

Fig. 1. Deflection of beam (constant magnitude) for fixed $F M=40000000, N=500000$ and various values of Ro.

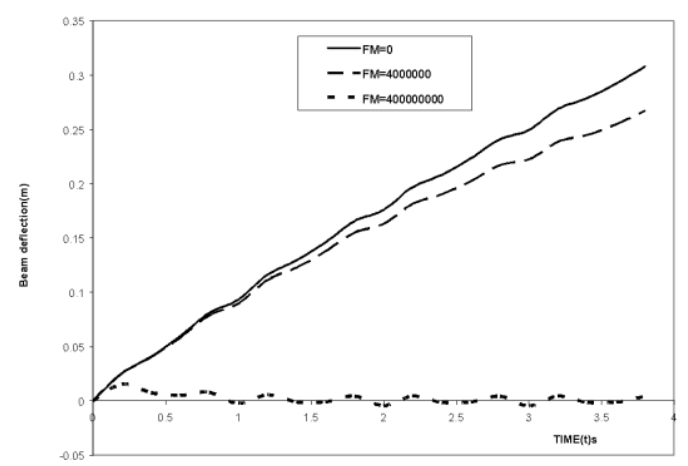

Fig. 2. Deflection of beam (constant magnitude) for fixed $R=0.5, N=500000$ and various values of $F M$.

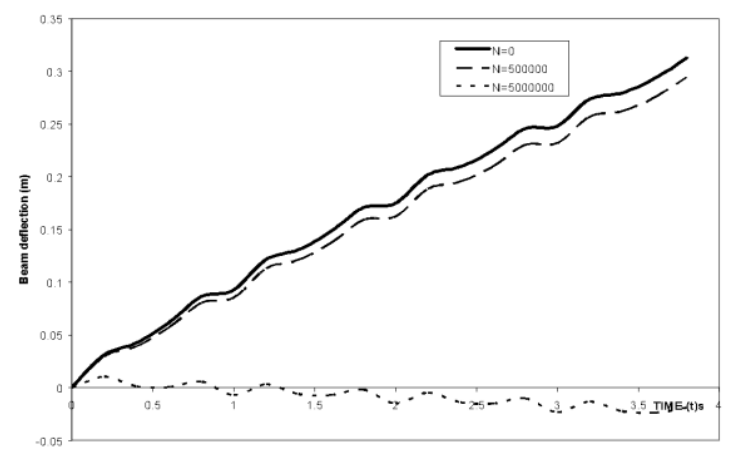

Fig. 3. Deflection of beam (constant magnitude) for fixed $R o=0.5, F M=4000000$ and various values of $N$

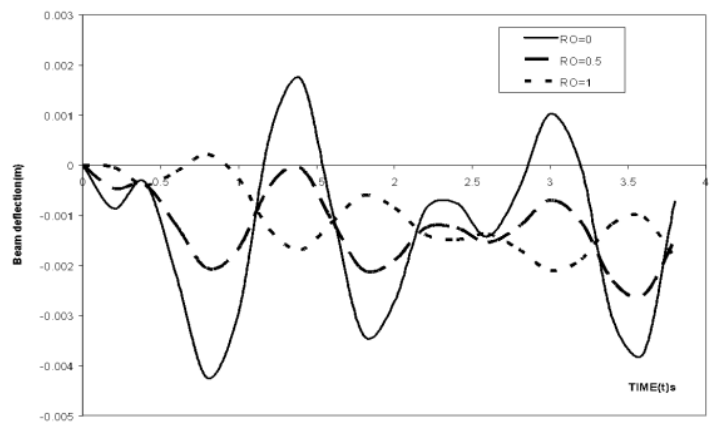

Figure 4. Displacement response of beam (harmonic magnitude) for fixed $\mathrm{FM}=\mathbf{4 0 0 0 0 0 0}, \mathrm{N}=500000$ and various values of $R$.

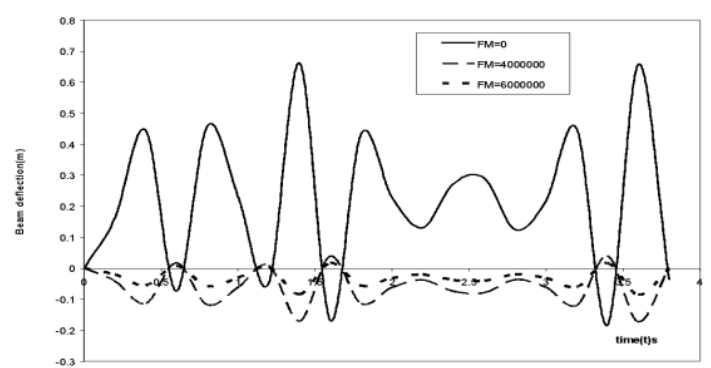

Figure 5. Displacement response of beam (harmonic magnitude) for fixed $\mathrm{Ro}=\mathbf{0 . 5}, \mathrm{N}=500000$ and various values of FM.

Published By: 


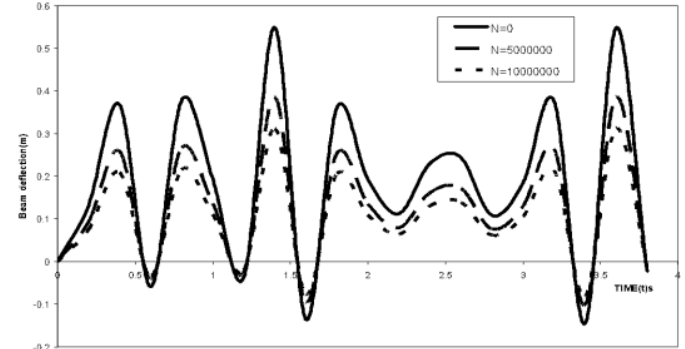

Figure 6. Displacement response of beam (harmonic magnitude) for fixed $F M=4000000, R o=0.5$ and various values of $\mathbf{N}$.

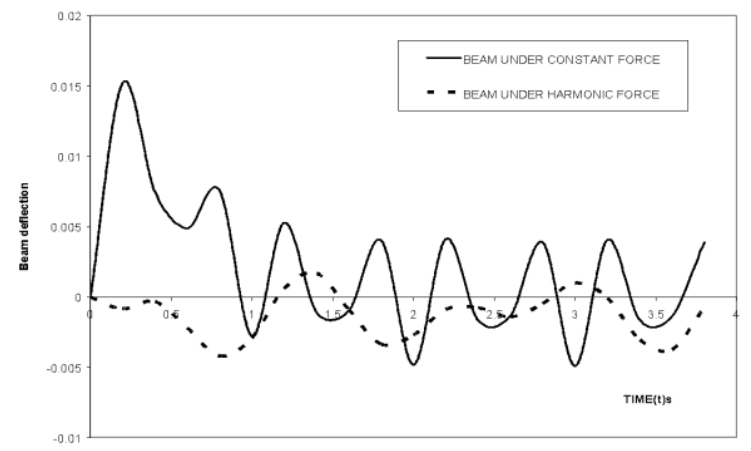

Figure 7. Comparison deflection of the beam on exponentially decaying foundation for constant and harmonic magnitude.

\section{CONCLUSIONS}

The problem of the vibration procedure of prestressed nonuniform beam having exponential decaying subgrade under mobile concentrated forces for both constant and harmonic variable forces is considered is examined. In this present paper. Solutions have been provided for the motion equation by the help of Galerkin's method which reduced it to second order ordinary differential equation. Numerical analysis for both constant and harmonic variable mobile forces is presented and various curves displayed.

It is observed that:

(i) as the Ro increases, the deflection of beam under the action of mobile concentration forces with constant velocity decrease for both constant and harmonic magnitude loads.

(ii) when the rotatory inertia $r_{0}$ is fixed, the amplitude of an exponential decaying foundation beam and traversed by concentrated forces travelling with constant speed decreases as the foundation modulus increases for both constant and harmonic magnitude loads.

(iii) increase in values of $\mathrm{N}$ reduce the deflection profile of non-prismastic beams for mobile concentration forces with constant velocity

\section{REFERENCES}

1. Saving, E; Dynamic amplification factor and response spectrum for the evaluation of Sound and Vibration. Vol. 248(2), 267-288, 2001.

2. Gbadeyan J.A. and Oni S.T. Dynamic Behaviour of Beams and Rectangular Plates under moving Loads. Journal of Sound and Vibration, 182(5), 677-695, 1995
3. Eisenberger, M., Yankelevsky, D Z., and Clastonic, J. Stability of beams on elastic foundation. Computers \& Structures, vol. 24, no. 1, pp. 135-139, 1986.

4. Kolousek, et al. Civil Engineering Structures subjected to Dynamic loads (in Slovak) SVTL, Bratislava. 1967.

5. Sadiku S. and Leipholz H. H. E. On the Dynamics of Elastic System with moving concentrated masses. Ingenieur Achives, 57, 223-242, 1989.

6. Knowles J. K. On the Dynamic Response of a beam to a Randomly moving load. Journal of Applied Mechanies 35. No.1, 1-6. 1968.

7. Gutierrez, R. H and Laura, A. A. Vibration of a beam of non-uniform cross-section traversed by a time varying concentrated force. Journal of Sound and Vibration, 207 (3), 419-425, 1997. http://dx.doi.org/10.1006/jsvi.1997.1164

8. Masoud Asgari. Vibration interaction analysis of non-uniform cross section beam under moving vehicle. International journal of Acoustics and vibration. Vol. 21, No 4, pp 429-439, 2016.

9. Ogunyebi S. N., Adedowole A., Fadugba S. E. and Oyedele E. A. The dynamic response of thin beam resting on variable elastic foundation and traversed by mobile concentrated forces Asian Journal of Mathematics and Computer Research 6(2): 181-192, 2015.

10. Frybal L. Non-Stationary response of a beam to a moving random force. Journal of Sound and Vibration 46, 323-338, 1976.

\section{AUTHORS PROFILE}

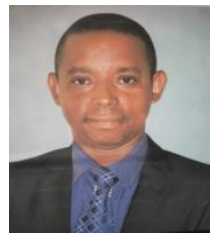

Dr. S. N. Ogunyebi, is a Senior Lecturer in the Department of Mathematics, Ekiti State University, Ado-Ekiti, Nigeria. He received his PhD from Ferderal University of Technology, Akure, Nigeria. E-mail address is segun.ogunyebi@eksu.edu.ng. His research interest is in Applied Mathematics He has published several research articles in both international and national Journals.

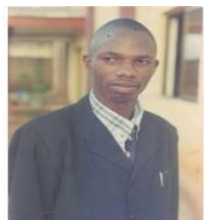

Dr. Adedowole A., Is a Senior Lecturer in the Department of Mathematical Sciences, Adekunle Ajasin University, Akungba, Akoko, Nigeria. His research interest is in Applied Mathematics He has published several research articles in both international and national Journals.

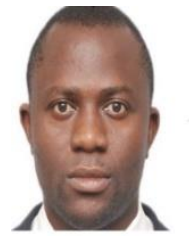

Ogunlade Temitope Olu, is a Lecturer in the Department of Mathematics, Ekiti State University, Ado-Ekiti, Nigeria. He Obtained his B.Sc. (Hons) degree (Mathematics) in 2004 at the University of AdoEkiti (Now Ekiti State University Ado-Ekiti) and proceeded to the University of Ibadan for his M. Sc. degree (Mathematics) in 2009. He later bagged a PhD degree (Mathematics) in 2016 at the Ekiti State University, Ado-Ekiti. His E-mail address is temitope.ogunlade@eksu.edu.ng. His research interest include, Applied Probability theory, Queueing Processes and Mathematical statistics. He has published several research articles in both international and national Journals of repute.

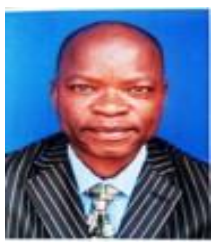

Oyedele, A. Akinola, $\mathrm{PhD}$ (Applied Geophysics) Lectures in the Department of Physics, Ekiti State University, Ado - Ekiti, Nigeria. Research interests are centered on Environmental / Engineering Geophysics/ Geotechnics. These include works on integrated geophysical/geotechnical subsoil characterization, structural earth works, soil stabilization / improvement, geoenvironmental monitoring and sustainability, challenges in groundwater development/contamination, instrumentation, resource recovery and Integrated GIS-Remote Sensing. 